\title{
The Association between Activity of Daily Living and the Combination of Alzheimer's Disease and Cataract in Elderly Requiring Nursing Care
}

\author{
Toshio Hamagishi' ${ }^{1}$, Toshimitsu Inagawa ${ }^{1}$, Yasuhiro Kambayashi ${ }^{1}$, Hiromasa Tsujiguchi ${ }^{1}$, \\ Masami Kitaoka1, Junko Mitoma', Hiroki Asakura1, Fumihiko Suzuki', Daisuke Hori', \\ Enoch Olando Anyenda', Nguyen Thi Thu Thao', Yuri Hibino', Koichi Hayashi², \\ Aki Shibata ${ }^{3}$, Takiko Sagara ${ }^{4}$, Jiro Okochi ${ }^{5}$, Kiyoshi Takamoku${ }^{5}$, Kotaro Hatta6, \\ Tadashi Konoshita7, Hiroyuki Nakamura' ${ }^{1}$

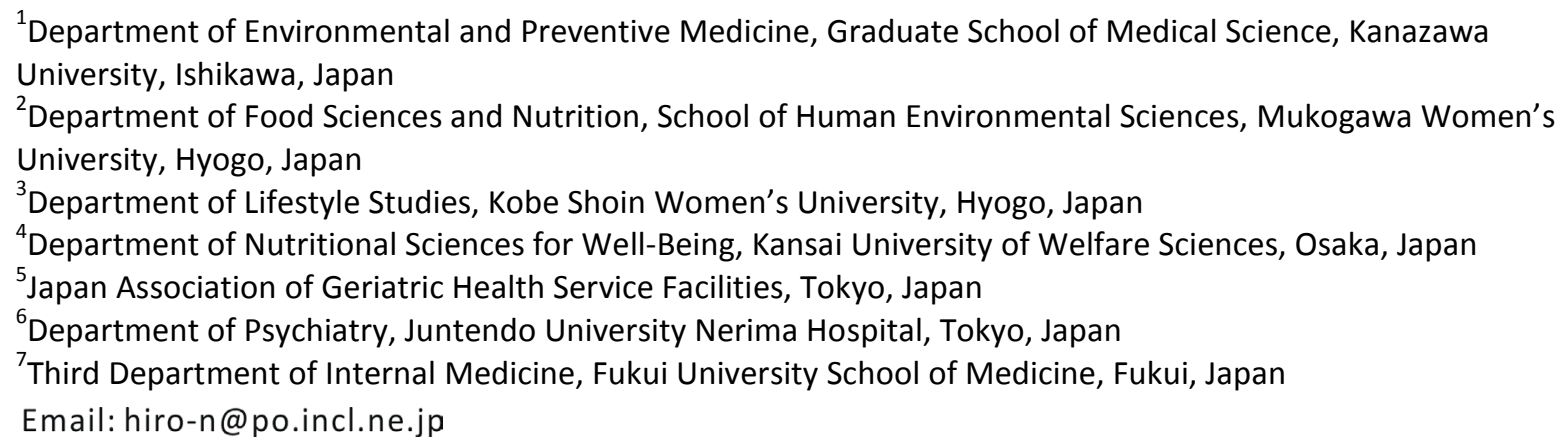

Received 16 June 2016; accepted 19 July 2016; published 22 July 2016

Copyright (C) 2016 by authors and Scientific Research Publishing Inc. This work is licensed under the Creative Commons Attribution International License (CC BY). http://creativecommons.org/licenses/by/4.0/

(c) () O) Open Access

\section{Abstract}

Some clinical and epidemiological studies have suggested that Alzheimer's Disease (AD) and cataract, may share common pathogenetic mechanisms, subsequently a positive association between the prevalence of $\mathrm{AD}$ and cataract, although other studies found no significant relationship between dementia and visual impairment including cataract in the elderly. Little is known about the association between Activity of Daily Living (ADL) and the combination of AD and cataract. To examine the association between ADL and the combination of AD and cataract, we performed a national survey in nursing care institutions in Japan, examining the decreased ADL in elderly with and without $A D$ and cataract for 453 elderly aged $85.0 \pm 8.13$ years. The proportion, $43.5 \%$ of $A D$ in subjects without cataract was significantly higher than that, $23.5 \%$ with cataract. Almost all ADL in AD with cataract was significantly lower than that without cataract, although all ADL in cataract 
patients receiving surgery in AD was significantly (all $p<0.002$ ) higher than that in cataract patients without surgery in $\mathrm{AD}$, as was confirmed by the multiple regression analysis incorporating into the relevant factors as independent variables. These results showed that ADL scores including cognitive functions decreased by cataract were increased by the surgery in the patients with AD. A significantly negative association between $A D$ and cataract seen in our data, which was inconsistent with the previous result, might lead to the necessities of the clinical diagnosis for slight severity of cataract for patients with AD.

\section{Keywords}

Alzheimer's Disease, Cataract, Elderly, Activity of Daily Living, Cognitive Function

\section{Introduction}

Alzheimer's Disease (AD) is the most common form of dementia. The number of individuals with AD has been increasing considerably in recent years, accounting for more than $50 \%$ of dementia cases [1]. AD causes the cognitive impairment of elderly in need of care, consequently interfering with daily tasks and decreasing Activities of Daily Living (ADL) in many ways [2]. Although epidemiologic studies have shown many risk factors such as occupational exposures (exposure to pesticides, electromagnetic fields, organic solvents and volatile anesthetics), pre-existing medical conditions (cerebrovascular disease, hypertension, diabetes, dyslipidemia, traumatic brain injury, depression and cancer) and lifestyle factors (smoking, consumptions of alcohol and coffee, body mass index, physical activity and cognitive activity) as non-genetic etiology of $\mathrm{AD}$ [3] [4], the accurate involvements of the occupational, pre-existing medical conditions and lifestyle factors in the development of $\mathrm{AD}$ remain to be elucidated.

It is estimated that there are 39 million blind persons and that cataract is the first cause of blindness worldwide [5]. Epidemiological studies have shown that smoking, diabetes mellitus, asthma, chronic bronchitis, cardiovascular disease, exposure to UVB light and corticosteroids use would increase cataract risk [6] [7]. Furthermore, over the last few years, clinical and epidemiological studies have suggested that dementia and visual impairment, in particular $\mathrm{AD}$ and cataract, may share common pathogenetic mechanisms [8]-[10]. Age-related vision impairment has been found to be closely associated with cognitive and behavioral manifestations [11]. Some epidemiologic studies also have demonstrated a positive association between AD and cataract [12]-[14]. Nevertheless, other studies found no significant relationship between cognitive impairment and vision loss including cataract in the elderly [15] [16]. In addition to the inconsistent studies on the association between AD and cataract, little is known about the association between Activity of Daily Living (ADL) and the combination of $\mathrm{AD}$ and cataract [17].

Both $\mathrm{AD}$ and cataract which increase with the aging deteriorate ADL and subsequently require nursing care [18]-[20]. To examine the association between ADL and the combination of AD and cataract, we performed a national survey in nursing care institutions in Japan, examining the decreased ADL in elderly with and without $\mathrm{AD}$ and cataract.

\section{Methods}

Fifty facilities were randomly selected out of 3410 nursing care institutions in Japan. Five resident patients and 5 daycare patients were sampled at random from each facility. A total of 500 patients without cerebral infarction, cerebral and subarachnoid hemorrhage were prospective subjects in this study. We mailed a questionnaire to a chief medical doctor in charge of each facility and subjects inquiring about ADL and medical conditions, including $\mathrm{AD}$ and cataract, as described below and obtained 453 responses (90.6\%, 273 resident patients and 180 daycare patients). This survey was conducted from 2010 to 2012. The average age and SD of the 453 subjects were 85.0 and 8.13 years old. This research was conducted after obtaining approval from the ethical committee of the Japan Association of Geriatric Health Service Facilities.

The questionnaires regarding medical conditions in subjects were related to $A D$ and cataract with and without surgery. To examine $\mathrm{AD}$, the questions concerned neuropsychological examinations including the patients' 
medical history, neurological testing, Mini-Mental State Examination [21], and standard clinical evaluation including brain scanning. In the diagnostic process, we used DSM-IV-TR and ruled out other factors. The National Institute of Neurological and Communicative Disorders and Stroke-Alzheimer's Disease and Related Disorders Association guidelines [22] were used for detection and diagnosis of either possible or probable AD, and stage of dementia according to the Clinical Dementia Rating (CDR) Scale [23]. We categorized as AD all those with CDR scale scores of 0.5 or higher also meeting the criteria for probable or possible AD. Cataract was diagnosed according to the International Classification of Diseases (ICD), $10^{\text {th }}$ Revision (H25) by family practice medical doctor. Diabetes mellitus was diagnosis according to Japan Diabetes Society clinical diagnosis guideline by family practice medical doctor [24]. The diagnoses of AD and cataract were performed within one year prior to the contact with nursing care institutions for elderly in this study. Our subjects included 125 patients with possible or probable $\mathrm{AD}, 253$ and 108 patients with cataract treated with surgery and conservatively, respectively, and 52 elderly without AD or cataract.

Regarding ADL, the questions were developed on the basis of a standard described previously [19] [25] and [26]. They dealt with bed mobility, transfer and locomotion, cognitive function (orientation), cognitive function (communication), cognitive function (mental activity), eating (swallowing), eating (feeding), toilet use, and bathing for elderly requiring both residential nursing care systems and those for daycare. Each ADL was categorized into a five-point scale. In the case of bed mobility, for example, a score of 5 represents being able to stand on one's feet and maintain this posture; score 4: having difficulty maintaining a standing posture, but being able to transfer from one place to another in a sitting position; score 3: being unable to move in a sitting position, but being able to sit in a proper posture without support; score 2: having difficulty sitting in an upright posture, but being able to turn over on a bed; and score 1: not being able to turn over on a bed. In the case of eating (feeding), a score of 5 represents being able to eat well without any support of others; score 4: spilling food during eating; score 3: having difficulty eating by themselves, but being able to eat with support for their posture and position of the dish; score 2: being unable to eat without complete support; and score 1: being unable to eat with any support (receiving tube feeding). Thus, the requirement for more concentrated nursing care during eating for the elderly decreased the ADL score. A lower score for each ADL implies worse ADL. In Table 1, we show ADL and prevalence of $\mathrm{AD}$, cataract treated with surgery, and cataract treated conservatively according to the status of nursing care (residential and daycare).

Pearson's correlation coefficients of age showed weak but significant associations with bed mobility $(-0.154)$, transfer and locomotion (-0.222), cognitive function (orientation) $(-0.207)$, cognitive function (communication) $(-0.161)$, cognitive function (mental activity) ( -0.191$)$, eating (dysphagia) $(-0.176)$, eating (feeding) $(-0.105)$, toilet use $(-0.161)$, and bathing $(-0.112)$. Because of significant differences in proportions in terms of the sex and age between elderly with and without diseases in addition to significant correlations between age and ADL scores, we compared each ADL score by analysis of covariance (ANCOVA) with age and sex as covariants, in which age was assigned as a continuous variable and sex were dummy-coded with follows: male 0 , female 1 . Mean ADL score and standard deviation adjusted by age and sex were calculated using regression coefficients corresponding to age, sex, and disease obtained by ANCOVA and raw mean values of age and sex in total subjects. To assess the interaction for ADL score between the combination of AD and cataract treated with or without surgery, we used two-way ANCOVA with age and sex as covariants. To confirm the results of ANCOVA, we performed multiple regression analysis for each ADL score incorporating into facility, sex, age, DM, cataract surgery, and Clinical Dementia Rating (CDR) scale as independent variables. The statistical software SPSS ver. 21 was used. P-values less than 5\% with two tails were considered statistically significant.

\section{Results}

Table 1 shows numbers (proportion) and age of residential or day care users with and/or without cataract and $\mathrm{AD}$ in this study. The proportion, $43.5 \%$ of $\mathrm{AD}$ in subjects without cataract were significantly higher than that, 23.5\% with cataract (Chi square test, $\mathrm{p}<0.000$ ). The proportions of woman using residential was significantly higher than that using day care. The proportions, $85.5 \%$ of women in subjects with AD were significantly higher than that, $66.1 \%$ without AD ( $p=0.000)$. The proportions, $69.6 \%$ of women without cataract was significant lower than that, $79.5 \%$ with cataract, respectively $(p=0.042)$ (Table 1$)$.

Table 2 shows that ADL adjusted by age and sex according to AD and cataract. The two-way ANCOVA showed statistically significant main effects of AD on ADL scores of cognitive functions including orientation, 
Table 1. Numbers (proportion) and age of residential or day care users with and/or without cataract and AD in this study.

\begin{tabular}{|c|c|c|c|c|}
\hline Facility & Cataract & AD & Proportion of women (\%) & Age (years; mean \pm SD) \\
\hline \multirow{4}{*}{ Residential } & \multirow{2}{*}{$(-) 53$} & $(-) 23$ & 17 (73.9) & $87.13 \pm 8.45$ \\
\hline & & $(+) 30$ & $23(76.7)$ & $84.90 \pm 6.06$ \\
\hline & \multirow{2}{*}{ (+) 220} & (-) 151 & $132(87.4)$ & $86.78 \pm 7.97$ \\
\hline & & $(+) 69$ & $60(87.3)$ & $86.55 \pm 7.49$ \\
\hline \multirow{4}{*}{ Day care } & \multirow{2}{*}{$(-) 39$} & $(-) 29$ & 15 (51.7) & $77.83 \pm 10.45$ \\
\hline & & $(+) 10$ & $9(90.0)$ & $81.50 \pm 6.50$ \\
\hline & \multirow{2}{*}{ (+) 141} & (-) 125 & $84(67.2)$ & $83.65 \pm 7.56$ \\
\hline & & $(+) 16$ & $11(68.8)$ & $84.25 \pm 6.98$ \\
\hline \multicolumn{5}{|c|}{ Statistics ( $p$ value) } \\
\hline Facility & ns & 0.000 & 0.000 & 0.000 \\
\hline Cataract & - & 0.000 & 0.042 & 0.010 \\
\hline AD & - & - & ns & ns \\
\hline
\end{tabular}

The proportions of AD in subjects without and with cataract showed $43.5 \%$ and $23.5 \%$, respectively $(\mathrm{p}<0.000)$. The proportion of women using residential and day use showed $85.5 \%$ and $66.1 \%$, respectively $(\mathrm{p}<0.000$ ). The proportion of women without and with cataract were $69.6 \%$ and $79.5 \%$, respectively $(\mathrm{p}=0.042)$.

communication and mental activity, eating including swallow and feeding, toilet use and bathing in addition to significant main effects of cataract on all ADL scores but bating. Furthermore, there were significant interactive effects of $\mathrm{AD}$ and cataract on ADL of transfer and locomotion, cognitive functions including orientation and communication, and toilet use (Table 2).

Age and sex-adjusted ADL in 361 cataract subjects with and without AD and surgery against cataract are shown in Table 3. There were statistically significant main effects of AD on ADL scores of cognitive functions including orientation, communication and mental activity in addition to significant main effects of surgery on all ADL scores. Furthermore, there were significant interactive effects of AD and surgery on ADL of transfer and locomotion (Table 3). Of 276 cataract subjects without AD, 228 (82.61\%) received cataract surgery, whereas only 25 (29.41\%) cataract surgery with AD of 85 cataract subjects, which difference in ratio of receiving surgery between patients with and without $\mathrm{AD}$ was recognized to be statistically significant by Chi-square test (p < 0.001).

Table 4 shows results on multiple regression analysis for each ADL score using facility, sex, age, DM, cataract surgery, and CDR score as independent variables in 356 subjects with cataract. Cataract surgery was statistically positively associated with ADL score of bed mobility, cognitive function (orientation), cognitive function (communication), cognitive function (mental activity) and toilet use. CDR was statistically negatively associated with all ADL scores but transfer and locomotion. There was no association of DM with any ADL score examined in this study (Table 4).

To summary these results, almost all ADL in AD with cataract was significantly lower than those without cataract, although all ADL in cataract patients receiving surgery in AD was significantly higher than those in cataract patients without surgery in $\mathrm{AD}$, as was confirmed by the multiple regression analysis incorporating into the relevant factors as independent variables.

\section{Discussion}

The present study showed a higher prevalence of AD in subjects without cataract than those with cataract, demonstrating a significant negative association between $\mathrm{AD}$ and cataract. On the contrary, recent population studies have demonstrated a significant positive relationship between AD and cataract. Mandas et al. [27], who analyzed data from 1168 subjects aged 65 years or older admitted between 2006 and 2013 to the outpatients clinic of the geriatric care unit at the University of Cagliari and to the one at Santissima Trinità Hospital in Cagliari, showed a relationships between mild cognitive impairment, various forms of age-related dementia (Alzheimer’s 


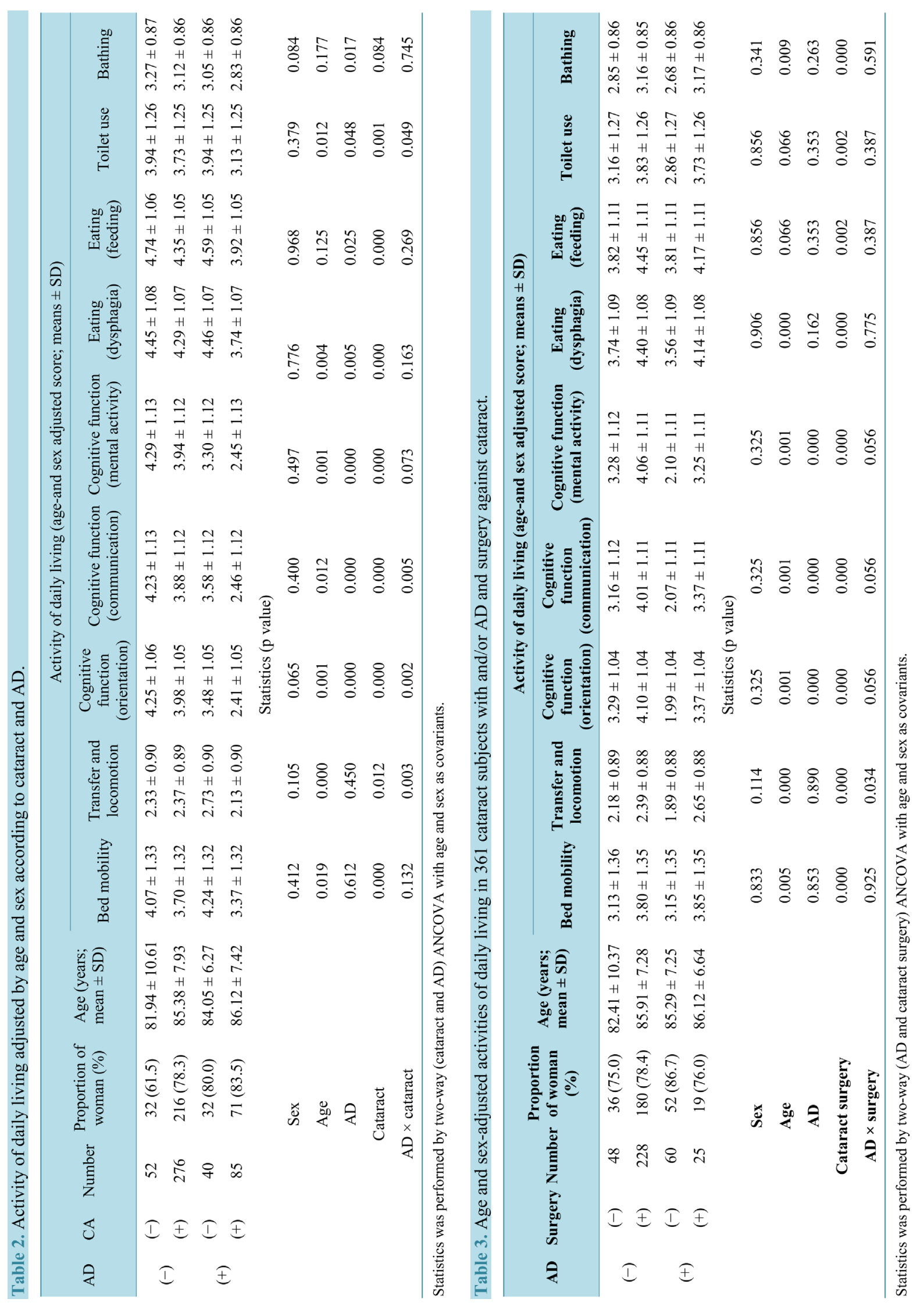




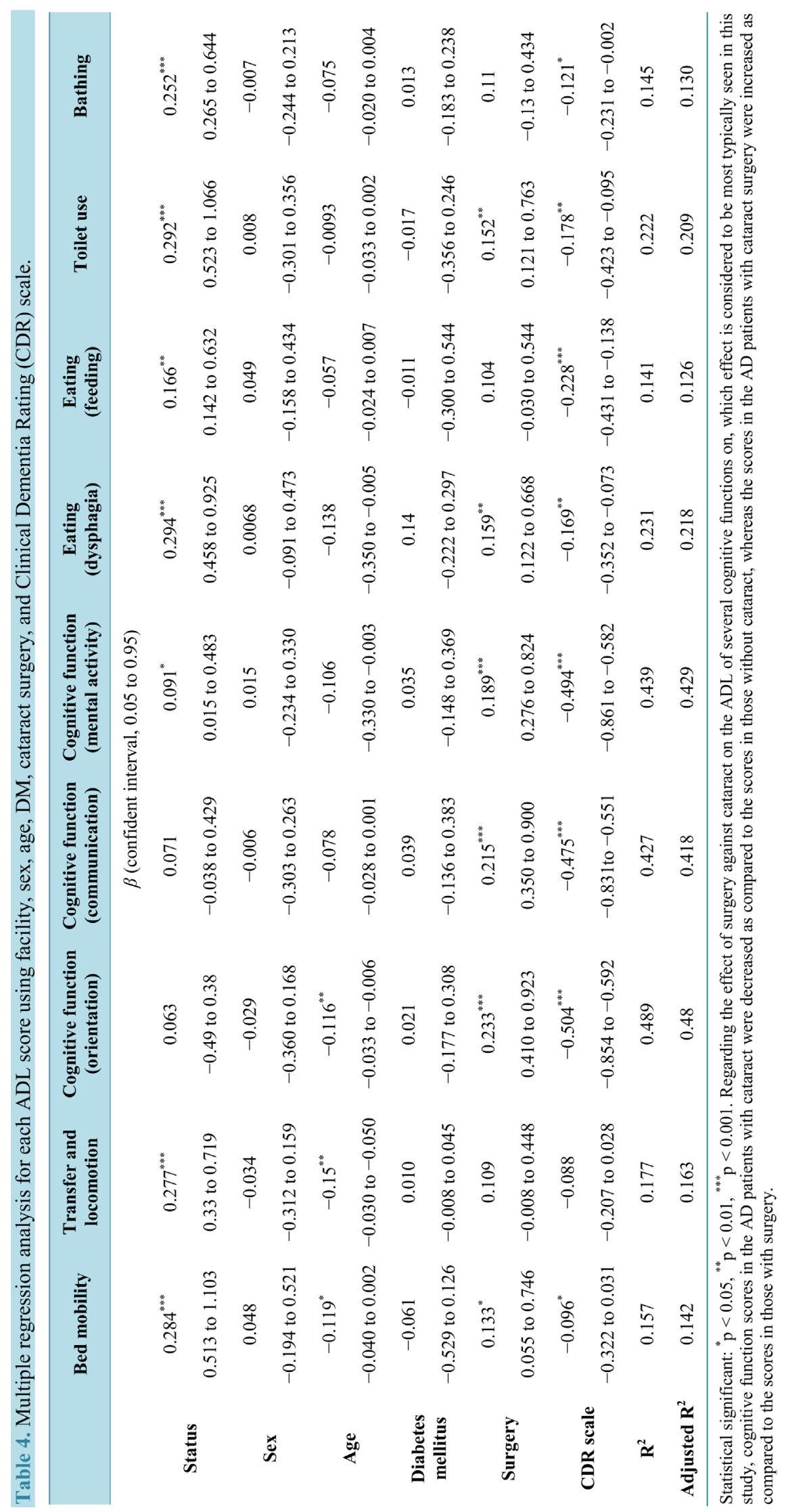


disease, mixed dementia, and vascular dementia) and the four major recognized age-related vision disorders, namely: cataract, age-related macular degeneration, glaucoma, and diabetic retinopathy. A population-based cohort study using data from Taiwan National Health Insurance Research Database including 491,226 subjects aged 70 or older with first-time diagnosis of cataract coded from 2000 to 2009 [13], showing that significantly lower rate of dementia in the cataract surgery group (hazard ratio $0.77 \%$, 95\% confidence interval $0.75-0.79$, p $<0.001$ ), concluded that patients undergoing cataract surgery were associated with a reduced risk of subsequent dementia compared with those without cataract surgery. A retrospective cohort study by using the database of the Taiwan National Health Insurance Program from 1999 to 2004 including 19,954 subjects aged 65 - 84 with newly diagnosed cataract showed that the adjusted HR of Alzheimer's disease was 1.43 (95\% CI 1.13, 1.82) for the cataract group, compared to the non-cataract group after adjustment for potential confounders, sex, age, diabetes mellitus, head injury and hypertension [14]. In contrast with these reports, cross-sectional analysis of data from the Newcastle 85+ Cohort study [12], comparing among no cataract, cataract diagnosis with and without surgery, found a lower degree of cognitive impairment in patients with cataracts, but no difference in cognitive impairment between cataract patients with and without surgery. The inverse results might be considered to be related to an enhanced help-seeking behavior in people with diagnosed cataract.

Our findings also do not support the theory of a combined neurodegenerative mechanism in cataract and cognitive impairment, as we found a negative association between cataract (including treated and untreated) and AD. Thus, our results agree well with Newcastle 85+ Cohort study [12], which was drawn from elderly subjects with almost the similar age as the mean age of 85.0 years in this study. Our study relied on family practice medical doctor for diagnoses of cataract in case of subjects without surgery. The association between AD and cataract for elderly subjects with very higher age might be different from that for relatively younger elderly. Very old elderly with $\mathrm{AD}$ could not be diagnosed to be cataract because they would not complain of visual loss even if they had cataract actually. This seems to be the reason why we had a negative association between AD and cataract in this study. As the diagnosis for cataract was performed based on examination for apparent changes of lens in this study, the cataract might be diagnosed only in case of cataract with the severe degree. In fact, the overall prevalence of cataract in this study showed $79.7 \%$, which is considered to be lower than $90 \%$ - $95 \%$ for subjects with the range of almost the same age [14]. Such a diagnostic bias accompanying AD, which has been pointed out by some studies [12] [14], might account for the negative association between AD and cataract. In fact, we recognized a very lower ratio of receiving cataract surgery in patients with AD as compared to those without $\mathrm{AD}$. The lower ratio of receiving surgery also seems to be very closely associated with lower prevalence of cataract in case of patients with AD. Epidemiology by Rogers and Langa [28] demonstrates that $77.9 \%$ of cataract patients with normal cognition had received at least one previous eye procedure as compared with $51.7 \%$ of those with $\mathrm{AD}$ for Americans aged 90 years or older. The under diagnosis for cataract with AD might explanation for our inverse association between cataract and AD. Therefore, our speculation would lead to the necessities of more accurate diagnosis for cataract in patients with AD.

It is noteworthy that ADL regarding cognitive functions with cataract surgery in AD patients was significantly higher than those without surgery, as was shown in the Table 3. Simultaneously, our results suggests that that cataract surgery improves cognitive function scores in the AD patients decreased by cataract to almost the same level as those in the subjects without AD. A number of studies examining the effect of cataract surgery on cognitive performance suggest that cognitive performance can be improved with cataract surgery [29] [30]. A prospective observational study performed by Tamura et al. for 20 patients with cognitive impairment showed that the grade of cognitive impairment in 12 patients (60\%) were made better after cataract surgery, concluding cataract surgery improved cognitive impairment in elderly Japanese patients [29]. However, a randomized study using a waiting list control group did not confirm this [31] [32]. Anstey et al. [31], who examined cataract surgery improve neuropsychological performance in healthy elderly using a randomized clinical trial of cataract surgery, showed no cognitive benefits of cataract surgery in cognitively normal adults, concluding that visual improvement following cataract surgery is not strongly associated with an improvement in neuropsychological test performance in elderly without cognitive impairment. Although there seems to be inconsistent results for the association between ADL regarding cognitive functions and cataract surgery in AD patients, our results are much different from other studies including Anstey et al. [31] [32] in the lights of age of the subjects and severity of AD. Furthermore, taken together with our results showing the multiple regression analysis controlling other relevant factors including AD severity confirmed our results, cataract surgery for highly aged elderly with AD seems to improve ADL regarding cognitive functions. The causal relationship between cataract surgery and 
the improvement of ADL in highly aged elderly with AD will be confirmed by the future follow-up study. In additions, we have some limitations regarding the outcome of cataract surgery depending on various factors; not assessing the association of cataract type with incidence of cognitive, not focusing on the first or second surgery and sequential surgery, not referring to one or both eyes, not examining analyzing the postoperative duration for each individual patient [30] [33].

\section{Conclusion}

In conclusion, our results showed that ADL scores including cognitive functions decreased by cataract were increased by the surgery in the patients with AD. Therefore, cataract surgery seems to improve ADL including cognitive functions for highly aged elderly with $\mathrm{AD}$. A significantly negative association between $\mathrm{AD}$ and cataract seen in our data, which was inconsistent with results from other studies, might lead to the necessities of the clinical diagnosis for slight severity of cataract for patients for AD.

\section{Acknowledgements}

The work was funded by a Grant-in-Aid for Scientific Research (B) from the Ministry of Health, Labour and Welfare (2011-2013), Japan.

\section{Conflict of Interest Statement}

None of the authors have any proprietary interests or conflicts of interest related to this submission.

\section{Limitations}

There are some limitations in our study. For example, our study population is small. Furthermore, we must assess the association of ADL by combination $\mathrm{AD}$ and cataract in long time. Also, we have conducted a survey using a questionnaire by mail. So, there may be individual differences by investigated staff. We cannot assess the association of cataract types with incidence of pathological change, not focusing on the detail of surgery. Further studies are needed to clarify the improvement of cognitive functions by cataract surgery in the patients with AD.

\section{References}

[1] Reiz, C., Brayne, C. and Mayeux, R. (2011) Epidemiology of Alzheimer Disease. Nature Reviews Neurology, 7, 137152. http://dx.doi.org/10.1038/nrneurol.2011.2

[2] Riccio, D., et al. (2007) Comprehensive Geriatric Assessment in Female Elderly Patients with Alzheimer Disease and Other Types of Dementia. Archives of Gerontology and Geriatrics, 44, 343-353. http://dx.doi.org/10.1016/j.archger.2007.01.047

[3] Jiang, T., et al. (2013) Epidemiology and Etiology of Alzheimer’s Disease: From Genetic to Non-Genetic Factors. Current Alzheimer Research, 10, 852-867. http://dx.doi.org/10.2174/15672050113109990155

[4] Reitz, C. and Mayeux, R. (2014) Alzheimer Disease: Epidemiology, Diagnostic Criteria, Risk Factors and Biomarkers. Biochemical Pharmacology, 88, 640-651. http://dx.doi.org/10.1016/j.bcp.2013.12.024

[5] Pascolini, D. and Mariotti, S.P. (2012) Global Estimates of Visual Impairment: 2010. British Journal of Ophthalmology, 96, 641-648. http://dx.doi.org/10.1136/bjophthalmol-2011-300539

[6] Abraham, A.G., Condon, N.G. and West Gower, E. (2006) The New Epidemiology of Cataract. Ophthalmology Clinics of North America, 19, 415-425.

[7] Prokofyeva, E., Wegener, A. and Zrenner, E. (2013) Cataract Prevalence and Prevention in Europe: A Literature Review. Acta Ophthalmologica, 91, 395-405. http://dx.doi.org/10.1111/j.1755-3768.2012.02444.x

[8] Goldstein, L.E., et al. (2003) Cytosolic $\beta$-Amyloid Deposition and Supranuclear Cataracts in Lenses from People with Alzheimer's Disease. The Lancet, 361, 1258-1265. http://dx.doi.org/10.1016/S0140-6736(03)12981-9

[9] Jun, G., et al. (2012) Delta-Catenin Is Genetically and Biologically Associated with Cortical Cataract and Future Alzheimer-Related Structural and Functional Brain Changes. PLOS ONE, 7, e43728. http://dx.doi.org/10.1371/journal.pone.0043728

[10] Tian, T., et al. (2014) Promise and Challenge: The Lens Model as a Biomarker for Early Diagnosis of Alzheimer's 
Disease. Disease Markers, 2014, Article ID: 826503. http://dx.doi.org/10.1155/2014/826503

[11] Clemons, T.E., Rankin, M.W. and McBee, W.L. (2006) Cognitive Impairment in the Age-Related Eye Disease Study: AREDS Report No. 16. Archives of Ophthalmology, 124, 537-543. http://dx.doi.org/10.1001/archopht.124.4.537

[12] Jefferis, J.M., et al. (2013) The Association between Diagnosed Glaucoma and Cataract and Cognitive Performance in Very Old People: Cross-Sectional Findings from the Newcastle 85+ Study. Ophthalmic Epidemiology, 20, 82-88. http://dx.doi.org/10.3109/09286586.2012.757626

[13] Yu, W.K. (2015) Cataract Suregery Is Associated with a Reduced Risk of Dementia: A Nationwide Population-Based Cohort Study. European Journal of Neurology, 22, 1370-1377. http://dx.doi.org/10.1111/ene.12561

[14] Lai, S.W., Lin, C.L. and Liao, K.F. (2014) Cataract May Be a Non-Memory Feature of Alzheimer's Disease in Old People. European Journal of Epidemiology, 29, 405-409. http://dx.doi.org/10.1007/s10654-014-9903-6

[15] Goldstein, F., Chen, J. and Hankinson, S.E. (2003) Cataract Extraction and Cognitive Function in Older Women. Epidemiology, 14, 493-497. http://dx.doi.org/10.1097/01.ede.0000083503.34133.8c

[16] Duron, E. and Hanon, O. (2008) Vascular Risk Factors, Cognitive Decline, and Dementia. Vascular Health and Risk Management, 4, 363-381.

[17] Zheng Selin, J., Orsini, N., Lindblad, B.E. and Wolk, A. (2015) Long-Term Physical Activity and Risk of Age-Related Cataract: A Population Based Prospective Study of Male and Female Cohorts. Ophthalmology, 122, 274-280. http://dx.doi.org/10.1016/j.ophtha.2014.08.023

[18] Marx, M.S., et al. (1995) Outcomes of Cataract Surgery in Nursing Home Residents. Psychosomatics, 36, $254-261$. http://dx.doi.org/10.1016/S0033-3182(95)71664-4

[19] Inagawa, T., et al. (2013) Decreased Activity of Daily Living Produced by the Combination of Alzheimer's Disease and Lower Limb Fracture in Elderly Requiring Nursing Care. Environmental Health and Preventive Medicine, 18, 680685. http://dx.doi.org/10.1007/s12199-012-0283-9

[20] Harrer, A., Gerstmeyer, K., Hirnschall, N., Pesudovs, K., Lundström, M. and Findl, O. (2013) Impact of Bilateral Cataract Surgery on Vision-Related Activity Limitations. Journal of Cataract \& Refractive Surgery, 39, 680-685. http://dx.doi.org/10.1016/j.jcrs.2012.11.028

[21] Folstein, M.F., Folstein, S.E. and McHugh, P.R. (1975) "Mini-Mental State”. A Practical Method for Grading the Cognitive State of Patients for the Clinician. Journal of Psychiatric Research, 12, 189-198. http://dx.doi.org/10.1016/0022-3956(75)90026-6

[22] McKhann, G., Drachman, D., Folstein, M., Katzman, R., Price, D. and Stadlan, E.M. (1984) Clinical Diagnosis of Alzheimer's Disease: Report of the NINCDS-ADRDA Work Group under the Auspices of Department of Health and Human Services Task Force on Alzheimer’s Disease. Neurology, 34, 939-944.

[23] Hughes, C.P., Berg, L., Danziger, W.L., Coben, L.A. and Martin, R.L. (1982) A New Clinical Scale for the Staging of Dementia. The British Journal of Psychiatry, 140, 566-572.

[24] Matsushima, M. (2002) [Japan Diabetes Society Clinical Practice Guideline]. Nihon Rinshō Geka Igakkai Zasshi, 60, 161-166

[25] Okochi, J., Utsunomiya, S. and Takatoshi, T. (2005) Health Measurement Using the ICF: Test-Retest Reliability Study of ICF Codes and Qualifiers in Geriatric Care. Health and Quality of Life Outcomes, 3, 46.

[26] Okochi, J., Takahashi, T., Takamuku, K. and Escorpizo, R. (2013) Staging of Mobility, Transfer and Walking Functions of Elderly Persons Based on the Codes of the International Classification of Functioning, Disability and Health. BMC Geriatrics, 13, 16.

[27] Mandas, A., et al. (2014) Cognitive Impairment and Age-Related Vision Disorders: Their Possible Relationship and the Evaluation of the Use of Aspirin and Status in a 65 Years-and-Over Sardinian Population. Frontiers in Aging Neuroscience, 6, 309.

[28] Rogers, M.A. and Langa, K.M. (2010) Untreated Poor Vision: A Contributing Factor to Late-Life Dementia. American Journal of Epidemiology, 171, 728-735. http://dx.doi.org/10.1093/aje/kwp453

[29] Tamura, H., et al. (2004) Improvement in Cognitive Impairment after Cataract Surgery in Elderly Patients. Journal of Cataract \& Refractive Surgery, 30, 598-602. http://dx.doi.org/10.1016/j.jcrs.2003.10.019

[30] Gray, C.S., Karimova, G., Hildreth, A.J., Crabtree, L., Allen, D. and O’Connell, J.E. (2006) Recovery of Visual and Functional Disability Following Cataract Surgery in Older People: Sunderland Cataract Study. Journal of Cataract \& Refractive Surgery, 32, 60-66. http://dx.doi.org/10.1016/j.jcrs.2005.07.040

[31] Anstey, K.J., et al. (2006) The Effect of Cataract Surgery on Neuropsychological Test Performance: A Randomized Controlled Trial. Journal of the International Neuropsychological Society, 12, 632-639. http://dx.doi.org/10.1017/S1355617706060954 
[32] Jefferis, J.M., Mosimann, U.P. and Clarke, M.P. (2011) Cataract and Cognitive Impairment: A Review of the Literature. British Journal of Ophthalmology, 95, 17-23. http://dx.doi.org/10.1136/bjo.2009.165902

[33] Mutoh, T., Isome, S., Matsumoto, Y. and Chikuda, M. (2012) Cataract Surgery in Patients Older than 90 Years of Age. Canadian Journal of Ophthalmology, 47, 140-144. http://dx.doi.org/10.1016/j.jcjo.2012.01.009

\section{Submit or recommend next manuscript to SCIRP and we will provide best service for you:}

Accepting pre-submission inquiries through Email, Facebook, Linkedin, Twitter, etc A wide selection of journals (inclusive of 9 subjects, more than 200 journals)

Providing a 24-hour high-quality service

User-friendly online submission system

Fair and swift peer-review system

Efficient typesetting and proofreading procedure

Display of the result of downloads and visits, as well as the number of cited articles

Maximum dissemination of your research work

Submit your manuscript at: http://papersubmission.scirp.org/ 